co: Source Implications and Health Risk. Atmosphere. 8(10): 196

Kline, C.J., Porcari, J.P., Hintermeister, R., et al. (1987) Estimation of from a one-mile track walk, gender, age and body weight. Med. Sports Exerc. 19:253-259.

Lecca, L.I., Campagna, M., Portoghese, I., et al. (2018). Work related stress, well-being and cardiovascular risk mo among nlight ternational Journal of Environmental Research and Public
Health. 15(9):1952.

Lohman, T.G., Roche, A.F., y Martorell, R. (1988). Anthropometric standardization reference manual (Vol. 177). Editorial Human kinetics books.

Pober, D.M., Freedson, P.S., Kline, G.M., et al. (2002). Development and validation of a one-mile treadmill walk test to predict peak oxygen uptake in healthy adults ages 40 to 79 years. Canadian Journal of Applied Physiology. 27(6):575-588.

Prioreschi, A., Brage, S., Westgate, K., et al. (2017). Cardiorespiratory fitness levels and associations with physical activity and body composition in young South African adults from Soweto. BMC public health. 17(1):301. https:// doi.org/10.1186/s12889-017-4212-0

Rikli y Jones (2001). Senior Fitness Test Manual. 2a ed. Editorial Human Kinetics.
Salvo, D., Villa, U., Rivera, J., et al. (2015). Accelerometerbased physical activity levels among Mexican adults an their relationship with sociodemographic characteristics and BMI: a cross-sectional study. International Journal of Behavioral Nutrition and Physical Activity. 12(79). https:/ doi.org/10.1186/s12966-015-0243-z

Secretaría de Salud. (2018). Encuesta Nacional de Salud y Nutrición 2018. Presentación de resultados. Disponible en: https://ensanut.insp.mx/encuestas/ensanut2018/doctos/ informes/ensanut_2018 presentacion_resultados.pdf

Triana-Reina, H.R., y Ramírez-Vélez, R. (2013). Asociación de la fuerza muscular con marcadores tempranos de riesgo cardiovascular en adultos sedentarios. Endocrinología y Nutrición. 60(8):433-438. Disponible en https: repository.usta.edu.co/bitstream/handle/11634/21017/505. pdf? sequence $=1$

Wilches-Luna, E.C., Hernández, N.L., Chavarro, P.A., et al. (2016). Cardiovascular risk profile and fitness in profes( Pública. 18(6):890-903.

, B. Levy, D. etal (1998). PreWison, P.W., D'Agostho, R.B., Lovy, D, et (1998).Prediction of col ries. Circulation. 97(18):1837-1847. Disponible en: https://
www.ahajournals.org/doi/pdf/10.1161/01.CIR.97.18.1837

\title{
Educación y capacitación para el trabajo: contexto penitenciario femenil de Nuevo León
}

\author{
Alicia González Cervantes*, Patricia Liliana Cerda Pérez*, Guadalupe
} Maribel Hernández Muñoz*

DOI:/https://doi.org/10.29105/cienciauanl25.106-2

\section{RESUMEN}

Para las mujeres privadas de la libertad, la formación educativa y capacitación de trabajo son aspectos clave en su preparación, al permitirles acceder a mejores oportunidades para ellas y sus familias, así como para desarrollarse personal y profesionalmente. Este artículo aborda resultados de la evaluación educativa y laboral de 60 de 308 internas del Centro de Reinserción Social Femenil de Escobedo, Nuevo León, durante 2019. Se encontró que tienen una expectativa proactiva por el alto grado de interés de seguirse formando y capacitando durante su proceso de reinserción social pero sin esta oportunidad se podrí propiciar el volver a deli peror.

Palabras clave: mujer y desarrollo, prisión, derecho a la educación, formación, educación inclusiva.

México se posiciona en el onceavo lugar del ranking de naciones en el informe de 2019 de la World Prison Brief, Institute for Crime \& Justice Policy Research (WPB), con un índice de 198,384 personas privadas de la libertad, de las cuales 10,591 eran mujeres. En 2018, el Instituto Nacional de Estadística y Geografia (Inegi) informa que la población penitenciaria en México era de 178,406 internos, $37.7 \%$ contaba con secundaria concluida, $32,4 \%$ preescolar o primaria y $14.7 \%$ preparatoria; $94.8 \%$ eran varones y $5.2 \%$ mujeres.

La educación, como derecho humano y punto clave del proceso de reinserción social que viven las personas privadas de la libertad dentro del centro penitenciario, es parte fundamental para el desarrollo y transformación personal de los internos. Como bien indica Freire (2005), al teorizar filosófica-

\section{ABSTRACT}

For women deprived of liberty, educational training and job training are key aspects in their preparation, allowing them to access better opportunities for themselves and their families and develop personally and professionally. This article addresses the results of the educational and employment evaluation of 60 of 308 inmates of the Escobedo of Nuevo León Center for Women's Social Reintegration during 2019; finding that they have a proactive expectation due to the high degree of interest in continuing their education and training during their social reinsertion process, but without this opportunity, reoffending could be propitiated.

Keywords: wo

mente, que la generación del conocimiento se vincula con la realidad de la persona, es decir que él mismo puede cerrarse y oprimirse ante su situación, sin embargo, seguir formándose podría romper con ese paradigma y transformar la vida de las internas proactivamente, al dar herramientas necesarias para reintegrarse a la sociedad y evitar así la reincidencia delictiva.

La Organización de las Naciones Unidas para la Educación, la Ciencia y la Cultura (UNESCO, 2020), precisa la educación como un derecho inclusivo en pro de transformar la vida para todos, a través del reforzamiento de la enseñanza técnica y la formación profesional. 
La Ley Nacional de Ejecución Penal de México (2016) dica que las personas privadas de la libertad tienen el derecho de seguir sus estudios básicos y de nivel medio superio gratuitamente de acuerdo a los programas y planes ofertados por el sector educativo, pero también una capacitación para el trabajo, como lo establece el Artículo 87, un proceso planeado y organizado para lograr la formación y el desarrollo de las habilidades y competencias para cumplir con las actividades laborales dentro del centro penitenciario.

La realidad de la mujer privada de la libertad se dificulta cuando llega a ser el pilar de la familia en todo sentido, por ello, en 2011, la Oficina de las Naciones Unidas en contra de la Droga y el Delito (UNODC) creó las Reglas de Bangkok, las cuales, en el Artículo 60, describen la necesidad de estructurar programas educativos y de capacitación para el trabajo, que les permitan, de acuerdo con su género, tener mejor oportunidad laboral, en actividades que les brinden confianza, desarrollo de las habilidades y competencias en torno a las áreas administrativas, de arte, cocina, peluquería y que les permitan ir preparándose para cuando sean liberadas.

Por lo anterior en este artículo se examinan los resultado el análisis de la realidad educativa y laboral que viven las mujeres privadas de la libertad, con el objetivo de explorar y describir, desde su punto de vista, las condiciones educativas y laborales que tienen como parte de su proceso de reinserción social, así como la proyección personal y profesiona que prevén tras su liberación al tener la oportunidad de seguir una formación educativa o de trabajo.

\section{MÉTODO}

Bajo el enfoque exploratorio-descriptivo, un esquema de investigación no experimental y transversal, el presente estudio se estructura a través del método cuantitativo, delimitado en obtener datos de la realidad del contexto educativo y labora que vive este gupo vinerable de mujeres privadas de laticocoso Escobedo, Nuevo León durante 2019.

a técnica que se utiliza es la encuesta realizada con e diseño de 18 reactivos o preguntas con el propósito de conocer, desde la perspectiva de las internas, la visión educativa y laboral durante su proceso de reinserción social. Se aplicaron 60 cuestionarios y posteriormente se realizó el análisis descriptivo utilizando SPSS, versión 25 , de cada una de las variables objetivo.

\section{Participantes}

La muestra está compuesta de 60 mujeres internas en su proceso de reinserción social dentro del Cereso de Escobedo, Nuevo León, que culminaron sus estudios de secundaria bajo firma de consentimiento informado por parte de las de la Secrón de Seguidad Pública del Estado, en abi de 2019, cundo se conta con 308 intens. Las mujes ences promedio de 31 ñ $(D E=73)$ l lo moyor fue 27 años; en los casos más jóvenes cuentan con 19 años y 49 años es la edad máxima; $68.3 \%$ de las encuestadas tiene entre 21 y 35 años.

Considerando el contexto del grupo vulnerable, la muestra fue dirigida como Hernández y Mendoza (2018) la describen, no probabilistice, al determinar que las enclest se aplicarín a quienes a dario y de for inform la seguridad de que se preservaría la estricta confidencialidad

RESULTADOS

\section{Contexto educativo de la mujer} privada de la libertad

El nivel educativo previo a la reclusión de las mujeres privadas de la libertad en el centro penitenciario se precisa en $36.7 \%$ de las 60 mujeres, las cuales, fuera del centro penitenciario, tuvieron la oportunidad de recibir una formación écnica (cursos prácticos) durante sus estudios de secundoria mientras 63.3\% no b recibió. El 50\% que recibió educación téci. desempion techica me dibujón tabilidad, electrónica y el taller de soldadura, en la figura 1 se muestra la distribución porcentual.

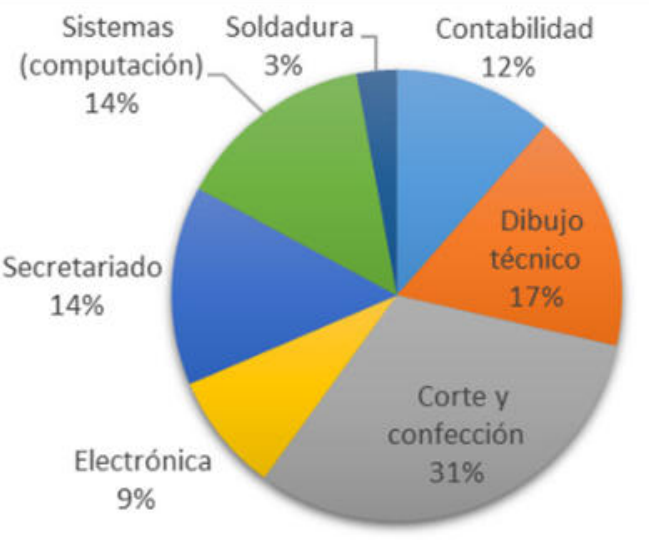

Figura 1. Educación técnica de las mujeres privadas de la libertad (elaboración propia)

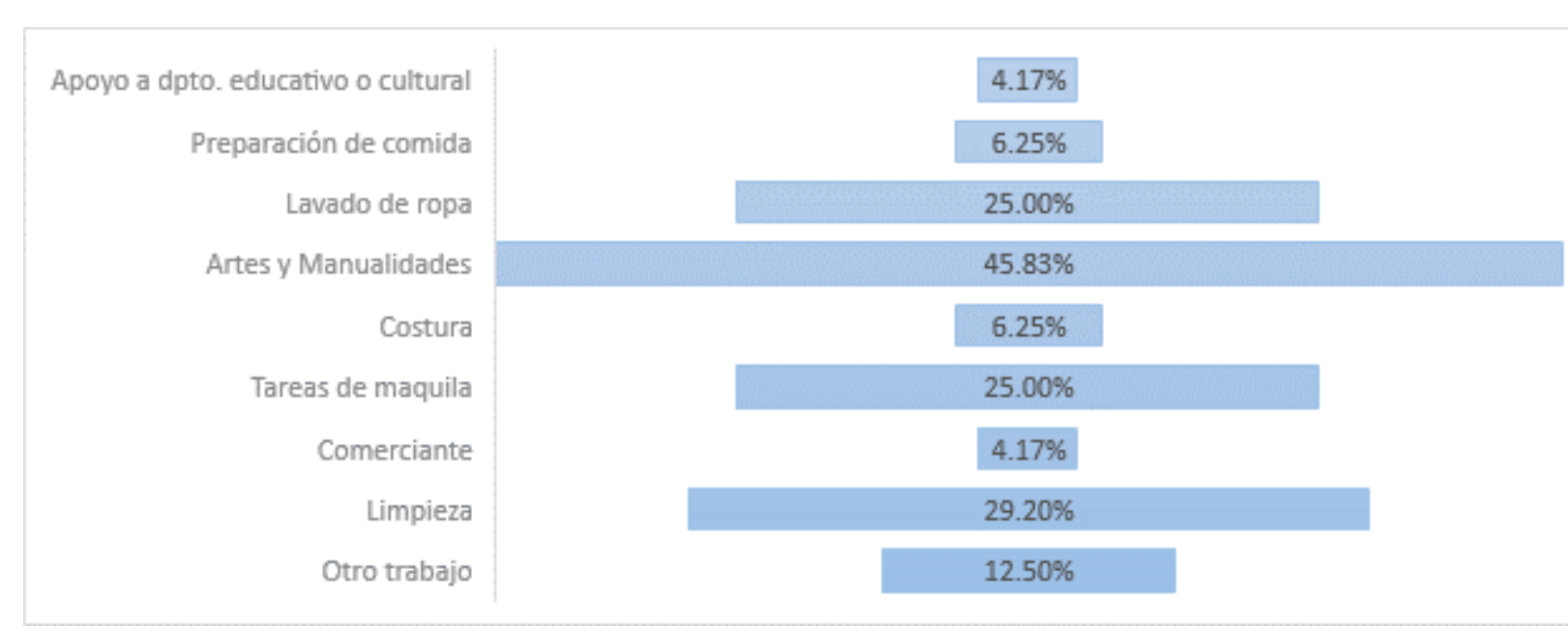

Figura 2. Actividades laborales de las mujeres privadas de la libertad (elaboración propia)

En el proceso de reinserción social de las mujeres interas, la oportunidad de tener una capacitación para el trabajo podría dar las herramientas necesarias para posicionarse en una mejor área laboral tanto dentro como fuera del Cereso, por lo tanto, de acuerdo con este análisis, se precisa que a $65 \%$ (39) de los 60 casos se le ofreció un curso con este fin, mientras a $35 \%$ no; de las 39 mujeres, $67 \%$ decidió de forma voluntaria ser partícipe de este proceso formativo. El $26.9 \%$ de internas realizó actividades vinculadas con manualidades y costura; $7.7 \%$ indicó que participaba en las relacionadas con la maquila de estropajos y velas; $3.9 \%$ en las de limpie$z$ a, preparación de comida y belleza, y $61.5 \%$ no especifico este dato.

\section{Participación laboral de las mujeres en el centro penitenciario}

Otro de los aspectos importantes en el desarrollo de las mujeres privadas de la libertad, en su proceso de reinserción, es oportunidad que se les ofrece dentro del centro penitenciario para realizar un trabajo; la mayoría ha decidido ocuparse en rabajar voluntariamente, generando un ingreso para ellas y sus familia mientras $20 \%$ no tuvo esta prico las que generan autoempleo se perfilan las de lavado de ropa. costura y concion penitenciario. En la figura 2 se especifican actividades laborales realizadas por las 48 internas.

\section{Realidad y proyección de las mujeres PL}

Centrándose en la evaluación de la posibilidad del seguimiento de una educación técnica en servicios (con capacitación en conpetencias profesionales laborales), como la que oferta la UANL, $95 \%$ de las 60 mujeres privadas de la libertad considera que les gustaria tener la oportunidad de seguir este tipo de formación durante su proceso de reinserción social dentro del centro penitenciario, para ir preparándose para cuando sean liberadas. Los resultados se muestran en la tabla I, donde se presentan, de acuerdo a la escala Likert (donde es muy interesante y 5 nada interesante), las actividadescon mayor intés, entre est́s destacan el diseño desalo mayor inets, ente 5 ms disto 
ción de alimentos y el corte de cabello, su cuidado ( $M=1.93)$, cambio de forma y de color, creación de maquillajes y peinados con una evaluación ( $\mathrm{M}=2.03)$.

En cuanto a la competitividad laboral, $100 \%$ de las mujeres que participaron en este estudio indicó que tendría mayores oportunidades al estar preparadas. Destacan las ventajas competitivas laborales, es decir, si contaran con una formaTabla I. Interés en realizar las actividades vinculadas a la formació educativa en servicios.

\begin{tabular}{|l|c|c|}
\hline \multirow{2}{*}{ Actividades } & \multicolumn{2}{|c|}{ Muy interesante } \\
\cline { 2 - 3 } & $\%$ & $f$ \\
\hline $\begin{array}{l}\text { Entrenamientos deportivos, sesiones } \\
\text { de educación y activación fisica, or- } \\
\text { ganización de coreografías y eventos } \\
\text { deportivos. }\end{array}$ & 41.7 & 25 \\
\hline $\begin{array}{l}\text { Preparación de alimentos (platillos, } \\
\text { bebidas, postres, productos de pana- } \\
\text { dería y repostería). }\end{array}$ & 46.7 & 28 \\
\hline $\begin{array}{l}\text { Programación, diseño y desarrollo de } \\
\text { sistemas y páginas Web, configura- } \\
\text { ción de equipos de cómputo. }\end{array}$ & 48.3 & 29 \\
\hline $\begin{array}{l}\text { Corte de cabello, cambio de forma y } \\
\text { tono, maquillajes, peinados, cuidado } \\
\text { depiel, pies y manos. }\end{array}$ & 43.3 & 26 \\
\hline $\begin{array}{l}\text { Diseño de espacios, producciones } \\
\text { (grá́ico, fotografía, medios audiovi- } \\
\text { suales, arquitectura, artes plásticas-di- } \\
\text { bujos). }\end{array}$ & 30 & 18 \\
\hline $\begin{array}{l}\text { Elaboración de patrones para confec- } \\
\text { cionar colecciones de ropa, a través de } \\
\text { las técnicas de bordado, drapeado. }\end{array}$ & 26.7 & 16 \\
\hline
\end{tabular}

*Elaboración propis

El $98.3 \%$ de las 60 internas encuestadas percibe que seguir una formación educativa de bachiller técnico podrí ser ventajoso para su preparación durante su proceso de reinserción social, además de ser necesario para su desarrollo personal, de acuerdo con $86.7 \%$ de las mujeres privadas de personal,
la libertad.

La expectativa de quienes cumplen una sentencia dentro del centro penitenciario femenil de Escobedo, Nuevo León en lo que respecta a su futuro laboral, coincide con los resultados de su interés formativo técnico, la mayoría (26.7\%) se visualiza trabajando en un restaurante, comedor o algun área donde se preparen alimentos; con un mismo porcentaje se posicionan las estéticas, peluquerías y clínicas de belleza y la capacitación; a 23.3\% le gustaría trabajar en una empresa u organización con actividades relacionadas con sistema computacionales y con el mismo porcentaje desean los cursos de esta área técnica.

Los principales hallazgos derivados de este estudio se vincutexto educativo y laborl que perfila expectativa de las mijeres privadas del Cento de Reisecín Socil de Escobedo jus en Nuvvo León. En chanto a aspecto sucalivo, cuatro de cada diez encuestadas contaban, previante a su reclusión con una formación técnica en secundaria, lo cual les permite y competencias en el seguimiento de su preparación en e proceso de reinserción social, así como identificar sus fortalezas en la preparación académica.

En lo que respecta a las habilidades laborales, ocho de cada diez mujeres que realizan algún trabajo dentro del centro penitenciario fortalecen su proceso para permititles hace conciencia de su realidad y de las fortalezs competic que pudiena surir de estas acciones Adenis, les pertive que pudieran surgir de estas acciones. Además, les permi les y las necesarias para cumplir las expectativas que tienede su futuro, y con ello evitar la reincidencia delictiva, permitiéndoles generar un plan de vida con un mejor ingreso económico.

La realidad que vive una persona privada de la libertad impacta en su vida personal, profesional y familiar, más au en el grupo vulnerable de internas, quienes, en la mayoría de los casos, son el pilar de a familia y tienen la mona da dese Ante ta situacion, las mujeres pivadas de la libertad consderan ventajoso seguir su formacion educativa para capacitarse y aprender algo útil para su preparación y así apoyar sus familiares, además de ocupar su tiempo proactivamente, a fin tener mejores hábitos que les ayuden a tener un bue historial en su expediente y esto redunde positivamente en considerar que para que este proceso se dé influyen muchos en los lineamientos jurídicos en pro del cumplimiento del derecho a la educacion en el sistema penitenciario, es pertinente diseñ Web, pa que, cur do

\section{profesional y familiar.}

\section{REFERENCIAS} tener conciencia de las bases para desarrollar las habilidades reflexionar en su realidad, así como en las acciones actuaAnde te su proceso de reinserción social; sin embargo, es importante factores internos y externos.
De los resultados obtenidos en el estudio y lo estipulado que se impulse la creación de convenios entre instituciones educativas públicas-privadas y autoridades penitenciarias para el diseño e implementación de programas aptos para las internas enfocados a capacitación de elaboracion deptí-

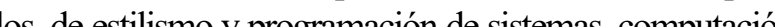
y diseno Web, par que,

Hernández, R., y Mendoza. P. (2018). Metodología de la Investigación, las rutas cuantitativa, cualitativa y mixta. Méxco:Mc Graw Hill Education.

Instituto Nacional de Estadística y Geografía. (2020). Censo Nacional de Gobierno, Seguridad Pública y Sistema pentenciario Estatales 2019. Presentación de resultados generales. Disponible en: https://www.inegi org mx/contenidos/ programas/cngspspe/2019/doc/cnospspe 2019 resultados. pdf

Organización de las Naciones Unidas para la Educación, Ciencia y la Cultura. (2020). La educación transforma vidas. Disponible en: https://es.unesco.org/themes/education World Prison Brief, Institute for Crime \& Justice Policy Research. (2019). World Prison Brief data. Disponible en Freire, P. (2005). Pedagogía del oprimido. México:Siglo https://www.prisonstudies.org/country/mexico 